\title{
USE OF TISSUE DOPPLER IMAGING TO DETECT RIGHT VENTRICULAR MYOCARDIAL DYSFUNCTION IN PATIENTS WITH CHRONIC OBSTRUCTIVE PULMONARY DISEASE
}

\section{JYOTHI SAMANTH ${ }^{1}$, PADMAKUMAR R ${ }^{2}$, ASHWINIKUMAR MOHAPATRA ${ }^{3}$, KRISHNANANDA N ${ }^{1}$, NAVIN PATIL ${ }^{4 *}$, KARTHIK RAO N ${ }^{5}$, VIDYA NAYAK ${ }^{1}$, BALAJI $0^{4}$, DIPANJAN ${ }^{4}{ }^{4}$, RAHUL KOTIAN ${ }^{6}$}

${ }^{1}$ Department of Cardiovascular Technology, School of Allied Health Sciences, Manipal University, Karnataka, India. ${ }^{2}$ Department of Cardiology, KMC Manipal, Manipal University, Manipal, Karnataka, India. ${ }^{3}$ Department of Pulmonary Medicine, KMC Manipal, Manipal University, Manipal, Karnataka, India. ${ }^{4}$ Department of Pharmacology, KMC Manipal, Manipal University, Manipal, Karnataka, India.

${ }^{5}$ Department of Medicine, KMC Manipal, Manipal University, Manipal, Karnataka, India. ${ }^{6}$ Department of Medical Imaging Technology, SOAHS, Manipal University, Manipal, Karnataka, India. Email: navin903@gmail.com

Received: 06 February 2017, Revised and Accepted: 10 March 2017

ABSTRACT

Objective: The objective of this study was to determine the utility of tissue Doppler imaging (TDI) in detecting early right ventricle (RV) myocardial dysfunction, given its prognostic implications in patients with chronic obstructive pulmonary disease (COPD).

Methods: A prospective case-control study was carried out involving 36 COPD patients in an acute exacerbated state as cases and 34 healthy subjects serving as controls. Each subject underwent a baseline echocardiography using various methods ranging from m-mode and two-dimensional measures for analyzing RV geometry to strain and strain rate using TDI to measure RV deformation. The cases underwent a subsequent echocardiogram 1 month later once the respiratory symptoms subsided.

Results: A significant difference was observed in RV tissue annular velocities (E', A', S) between cases and the controls at baseline. However, no significant increase was observed in tissue annular velocities among cases during remission states from baseline. Peak systolic strain in COPD group was significantly reduced in comparison to controls, but not significantly increased during remission when compared to baseline in cases. Forced expiratory volume in 1 second/vital capacity did not bear any significant correlation with RV strain. TEI index had a negative linear correlation with peak systolic strain of RV, which was statistically significant.

Conclusion: TDI parameters revealed that RV dysfunction remains unaltered even in remission state of COPD despite pulmonary arterial pressure normalizing. In light of it bearing a negative prognosis in COPD, RV dysfunction merits assessment in COPD patients, both in acute exacerbations as well as in remission.

Keywords: Strain rate, Tissue annular velocity, Doppler, Right ventricle geometry, Case-Control.

(C) 2017 The Authors. Published by Innovare Academic Sciences Pvt Ltd. This is an open access article under the CC BY license (http://creativecommons. org/licenses/by/4. 0/) DOI: http://dx.doi.org/10.22159/ajpcr.2017.v10i6.17517

\section{INTRODUCTION}

Chronic obstructive pulmonary disease (COPD) is associated with considerable morbidity and is predicted to become the third leading cause of death worldwide by $2020[1,2]$. Early in the course of the disease respiratory symptoms predominate, while later systemic complications include osteoporosis, loss of fat-free mass [3], and cardiovascular disease $[4,5]$. Cardiovascular complications in patients with COPD include cor pulmonale [6], a 2-3 fold excess cardiovascular event risk even after allowing for confounders such as smoking [7] and a greater than fourfold excess risk of chronic heart failure [8]. Arterial stiffness, an independent predictor of cardiovascular outcome $[9,10]$ was increased in patients with COPD, free of cardiovascular disease, suggesting acceleration of age-dependent stiffening [11]. It was found that even in mild severity airways obstruction, stiffening develops in early lung disease in parallel with asymptomatic changes in pulmonary arterial (PA) pressure and right ventricular (RV) function [12,13]. Long-term effects of increased arterial stiffness in COPD are unknown but might include cardiac adaptations similar to those in normal aging [14-17], systemic hypertension [18], and diastolic heart failure [19]. Such changes in cardiac structure and function may underlie the increased risk of cardiovascular disease in COPD.

COPD is often associated with changes of the structure and the function of the heart. Although functional abnormalities of the RV have been well described in COPD patients with severe hypoxemia, little is known about these changes in patients with normoxia and mild hypoxemia. The evaluation of RV function is clinically useful in patients with COPD because the presence of RV failure has prognostic implications [20,21]. All invasive and noninvasive techniques evaluating RV performance have important limitations due to the complex geometry of the RV. The introduction of Doppler measurement of myocardial wall velocities (tissue Doppler imaging (TDI) and the recent development of strain rate imaging (SRI) technique have made a more adequate assessment of global and regional systolic and diastolic RV function possible. Hence, the aim of this study is to detect early RV myocardial dysfunction in patients with COPD using TDI, and the main objectives are to compare the TDI parameters such as strain, SR, tissue annular velocities between COPD patients who are in exacerbation state and healthy individuals, $\mathrm{RV}$ deformation parameters in patients at exacerbation event and after the remission state, the TDI-derived parameters in COPD patients who are in remission state and healthy individuals and to assess the correlation among tissue Doppler parameters and pulmonary function tests in COPD patients.

\section{METHODS}

A prospective, case-control study, compliant with the Declaration of Helsinki, was performed in the Department of Cardiology, Kasturba Hospital, Manipal. Institutional Ethical Clearance was obtained. Subjects were recruited after obtaining a written informed consent. Patients with COPD in an acute exacerbation state were included as 
cases. Further, subjects with clinical evidence of cor pulmonale, clinical or electrocardiographic evidence of systemic hypertension, myocardial ischemia, valvular diseases and any irregular cardiovascular rhythm disorders were excluded from the study. Healthy subjects appearing for health check-up, who had normal cardiac findings served as controls and were drawn by frequency matching technique. Echocardiographic examination was performed before medical therapy was initiated. Subsequently, a comparison echocardiographic examination was performed at least 1 month after the symptoms were controlled. The diagnosis of COPD was made on the basis of a review of patient's history, physical examination, chest radiographs, and standard pulmonary function tests; according to the criteria established by the global initiative for chronic obstructive lung disease [22]. Acute exacerbation of COPD was defined as worsening dyspnea, increase in sputum purulence and increased sputum volume in accordance with ACCP acute exacerbation guidelines [23].

\section{Echocardiography}

Individuals were subjected to complete clinical examination and transthoracic echocardiography using Vivid 7 - echocardiography system with a $2.5 \mathrm{MHz}$ transducer. Echocardiographic parameters were evaluated after pulmonary function test was performed, i.e., forced expiratory volume in 1 second/vital capacity (FEV1/VC).

Measurements of right heart chambers were done according to established criteria [24,25]. RV end-diastolic (ED) and end-systolic (ES) areas were assessed by manual planimetry, and then RV fractional area change (RVFA) was derived using the formula [20].

Using m-mode technique RVED dimension (EDD), isovolumic contraction IVC diameter, and left ventricular (LV) internal dimensions were obtained. Tricuspid annular plane systolic excursion (TAPSE) was measured in $\mathrm{A} 4 \mathrm{CH}$ view. Trans tricuspid valve Doppler flow velocities, i.e., peak early and late diastolic velocities were recorded from $\mathrm{A} 4 \mathrm{CH}$ view, Deceleration time, isovolumic relaxation time (IVRT), IVC time (IVCT), and ejection time were measured with the additional recording of transpulmonary valve Doppler flow velocity. Pulmonary artery systolic pressure was estimated by continuous wave Doppler as 4 times square of peak regurgitation velocity plus assumed right atrial pressure of $10 \mathrm{mmHg}$ (fixed value method) [20]. For the analysis of global RV function, Doppler parameters were used to derive the TEI index, i.e., (IVCT+IVRT)/ET.

\section{TDI}

Recordings were stored digitally as two-dimensional (2D) cineloops and were transferred to an optical disk medium workstation for offline analysis. The images showing the tissue motion velocity were superimposed on the 2D echocardiographic images for real-time color display. TDI annular velocities during systole, early relaxation (Ea), and atrial systole (Aa) were possessed from RV free wall (FW) and interventricular septum (IVS) at basal site in the $\mathrm{A} 4 \mathrm{CH}$ view. The strain (change in length per unit length) in each segment is defined [26] as the relative magnitude of segmental deformation. From tissue Doppler data, SR was estimated by calculating the velocity gradient. The time integral of incremental SR yields logarithmic strain: E=log (L/LO). In this study, the logarithmic strain was converted to Lagrangian strain: $\mathrm{E}=(\mathrm{L}-\mathrm{L} 0) / \mathrm{L} 0$.

SR is determined during systole (S), early diastole (E), and late diastole (A). Systolic strain was measured from the same wall site in the same views. TDI wall velocities at the tricuspid annulus level during systole, early relaxation and atrial systole were also obtained.

\section{Statistical analysis}

Sample size was calculated to be 29 cases and 29 controls and statistical analysis was done. Data were entered in excel sheet and analyzed using appropriate statistical methods (independent t-test for intergroup comparison and paired t-test for intragroup comparison). A $\mathrm{p}<0.05$ was considered as statistically significant.

\section{RESULTS}

This study included 36 subjects in COPD with acute exacerbation group and 34 controls. The mean age in COPD group and controls were $63 \pm 9.9$ and $59 \pm 10$, respectively. Cases, in our study, included 16 females and 20 males (Table 1). Among the subjects with COPD 5 (13.9\%) were smokers and $3(8.3 \%)$ were alcoholic.

About 36 subjects with acute exacerbation of COPD underwent pulmonary function test as diagnostic sequence. The mean value of FEV1/VC) was found to be $0.62 \pm 0.11$ resembling obstructive in its nature. After a month of acute exacerbation (A/E), event subjects were enrolled for repeat PFT after confirming their remission state based on their symptoms. The test showed a significant increase in the value of FEV1/VC among subjects in remission state after an exacerbation event, i.e., $0.62 \pm 0.11$ vs. $0.67 \pm 0.12$ ( $p=0.001$ ) (Fig. 1).

Echocardiography was performed on COPD patients in their exacerbated state which included various methods ranging from m-mode and 2D measures for analyzing RV geometry to strain and SR using TDI to measure RV deformation. To ensure the subclinical as well as clinically significant changes that occur in COPD subjects, the echocardiographic parameters were compared with the healthy individuals who were matched with respect to age and gender.

Thus, echocardiographic parameters were compared between three groups which included patients with acute exacerbation of COPD, same subjects at remission state and the control group. The parameters among the patients with acute exacerbation of COPD state and in the remission state were analyzed using paired t-test ensuring that the continuous variable was normally distributed. The variables were compared with the controls using independent t-test.

\section{LV m-mode parameters}

LV m-mode echocardiography showed statistically significant difference in IVS dimension, LVEDD, ESD between COPD patients and controls with $\mathrm{p}=0.007,0.03$, and 0.002 , respectively. LVEF as measured by Simpson's method was significantly reduced in patients with COPD when compared with controls $(p=0.02)$ (Table 2).

\section{RV geometry}

RV geometry assessment was made by M-mode echocardiography, 2D RV dimension to obtain RVFW thickness, RVED dimension, TAPSE, RVFA change. According to the current study the RVFW thickness, RV

Table 1: Cases and controls

\begin{tabular}{llll}
\hline Group & \multicolumn{2}{l}{ Gender } & Total \\
\cline { 2 - 3 } & Male & Female & \\
\hline Controls & 11 & 23 & 34 \\
COPD subjects & 20 & 16 & 36 \\
Total & 31 & 39 & 70 \\
\hline
\end{tabular}

COPD: Chronic obstructive pulmonary disease

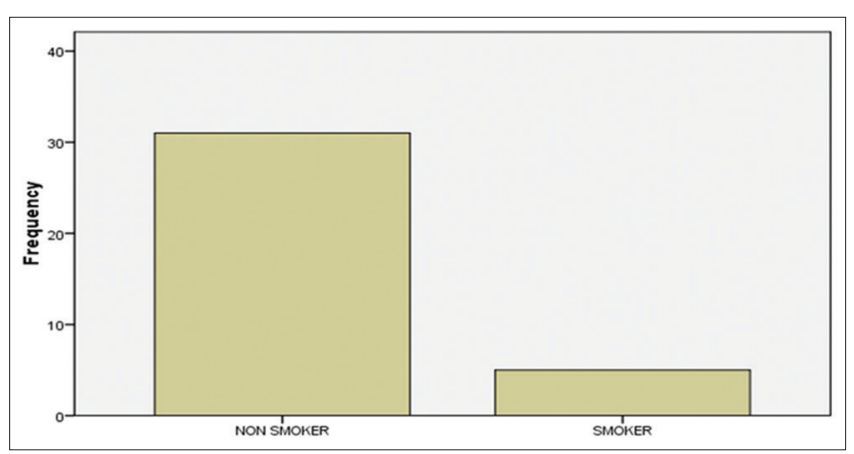

Fig. 1: Cases and control percentage distribution 
internal dimensions were found to be increased in COPD patients when compared with controls. There was statistically significant difference noted in the value of TAPSE between COPD patients and controls (Table 3). As far as RV geometry is considered, there was no significant change in the values of RV internal dimensions and FW thickness were noted in A/E COPD patients during the follow-up period in the remission state; however, the IVC dimension was significantly higher in COPD group and was found to be reduced in remission state from $\mathrm{A} / \mathrm{E}$ event.

\section{Doppler echocardiography}

Findings showed significantly elevated RV systolic pressure in COPD patients when compared with controls; however, this was found to be reduced after remission state of $A / E$ of $\operatorname{COPD}(p<0.001)$. Tricuspid late diastolic A wave, IVRT, TEI index values were significantly increased in COPD patients whereas E/A ratio, deceleration time were significantly reduced in COPD with $\mathrm{A} / \mathrm{E}$ group, whereas deceleration time was found to be improved after remission state $(p=0.01)$ (Table 4).

\section{TDI}

RV tissue annular velocities (E', A', S) were found to be significantly different between COPD subjects and the controls; however, RV E' and $S$ values were significantly reduced in COPD group with no significant increase in its value after the remission state; suggesting, the subtle decline in RV function parameters remain unchanged even in the remission state after an A/E event in COPD patients. E' and A' of IVS were also reduced in COPD patients, whereas S-Systolic annular velocity did not show significant difference among three groups. Reduction in diastolic component of tissue annular velocity of IVS, i.e., E' may be due to RV strain which is resulting in impaired relaxation of IVS (Table 5).

\section{RV deformation}

Imaging revealed a significant reduction in peak systolic strain in patients with A/E of COPD when compared with controls, and was slightly increased at the remission state on follow-up from the baseline, but found to be statistically insignificant. Peak systolic strain of IVS was reduced significantly among A/E group in comparison with controls, and the same in follow-up also showed increased value, suggesting improved IVS strain in remission state after an A/E event in COPD patients. Systolic SR (SSR) of both RVFW and the IVS were significantly reduced in COPD with A/E patients with no significant change in its value on follow-up noted. Early diastolic SR (ESR) in RVFW was lower in COPD group, whereas ESR of IVS did not show a significant difference between each group (Table 6).

Correlation between FEV1/VC and the tissue Doppler parameters was analyzed using Pearson correlation test depending on the normality

Table 2: ECHO parameters

\begin{tabular}{llll}
\hline Echo parameters & Controls & COPD with A/E state & Remission state after A/E event \\
\hline IVSD (mm) & $8.35 \pm 1.12$ & $9.28 \pm 1.59$ & $9.19 \pm 1.33^{*}$ \\
IVSS (mm) & $13.09 \pm 1.42$ & $13.53 \pm 1.92$ & $13.83 \pm 1.5$ \\
LVEDD (mm) & $42.94 \pm 7.87$ & $46.42 \pm 5.49$ & $46.36 \pm 5.53^{*}$ \\
LVESD (mm) & $26.21 \pm 3.86$ & $28.97 \pm 4.48$ & $29.08 \pm 3.67^{*}$ \\
PWD (mm) & $9.12 \pm 3.9$ & $8.33 \pm 1.21$ & $8.06 \pm 0.9$ \\
PWS (mm) & $12.53 \pm 2.7$ & $12.31 \pm 1.43$ & $12.36 \pm 1.07$ \\
EF (\%) & $70.15 \pm 3.47$ & $67.86 \pm 4.87^{*}$ & $67.89 \pm 3.7^{*}$ \\
FS (\%) & $39.53 \pm 2.85$ & $37.89 \pm 3.74^{*}$ & $37.83 \pm 2.48^{*}$ \\
HR (bpm) & $81.53 \pm 17.99$ & $86.89 \pm 14.93$ & $79.78 \pm 11.11^{\S}$ \\
\hline
\end{tabular}

IVSD: Inter ventricular septum in diastole, IVSS: Inter ventricular septum in systole, LVEDD: Left ventricular end diastolic dimension, LVESD: Left ventricular end systolic dimension, PWD: Posterior wall in diastole, PWS: Posterior wall in systole, EF: Ejection fraction, FS: Fractional shortening, HR: Heart rate, COPD: Chronic obstructive pulmonary disease, ${ }^{*} \mathrm{p}<0.05$ vs. controls, ${ }^{8} \mathrm{p}<0.05$ vs. A/E of COPD group

Table 3: RV geometry

\begin{tabular}{llll}
\hline Echo parameters & Controls & COPD with A/E state & Remission state after A/E event \\
\hline RVFWT (mm) & $3.95 \pm 0.65$ & $5.83 \pm 0.87^{*}$ & $5.91 \pm 1.02^{*}$ \\
RVEDD (mm) & $21.76 \pm 3.2$ & $23.28 \pm 4.19$ & $22.64 \pm 3.55$ \\
RVED SAX (mm) & $25.53 \pm 4$ & $26.14 \pm 4.91$ & $28.03 \pm 7.31$ \\
RVED LAX (mm) & $52.5 \pm 7.46$ & $52.83 \pm 7.24$ & $51.83 \pm 9.26$ \\
RVES SAX (mm) & $20.32 \pm 2.84$ & $23.00 \pm 5.86^{*}$ & $23.97 \pm 6.6^{*}$ \\
RVES LAX (mm) & $37.85 \pm 5.01$ & $40.11 \pm 6.28^{*}$ & $41.11 \pm 0.76^{*}$ \\
RVFA (\%) & $46.04 \pm 7.16$ & $38.48 \pm 9.38^{*}$ & $38.77 \pm 8.3^{*}$ \\
TAPSE (mm) & $21.88 \pm 2.38$ & $20.25 \pm 2.5^{*}$ & $20.19 \pm 2.14^{*}$ \\
IVC MAX (mm) & $12.44 \pm 1.44$ & $13.61 \pm 3.6$ & $12.50 \pm 3.1$ \\
IVC MIN (mm) & $5.44 \pm 89$ & $7.03 \pm 2.5^{*}$ & $6.22 \pm 2.21^{* \S}$ \\
\hline
\end{tabular}

RVFWT: Right ventricular free wall thickness, RVEDD: Right ventricular end diastolic dimension, SAX: Short axis, LAX: Long axis, RVED: Right ventricular dimension in end diastole, RVES: Right ventricular dimension in end systole, RVFA: Right ventricular fractional area change, TAPSE: Tricuspid annular plane systolic excursion, IVC: Inferior vena cava, MAX: Maximum, MIN: Minimum, COPD: Chronic obstructive pulmonary disease, RV: Right ventricle, *p<0.05 vs. controls, ${ }^{\S} \mathrm{p}<0.05$ vs. A/E of COPD group

Table 4: Doppler echocardiography

\begin{tabular}{llll}
\hline Echo parameters & Controls & COPD with A/E state & Remission state after A/E event \\
\hline RVSP (mmHg) & $22.79 \pm 3.51$ & $39.25 \pm 10.58^{*}$ & $29 \pm 5.3^{*}$ \\
TV-E (cm/s) & $62.68 \pm 13.83$ & $60.25 \pm 15.71$ & $59.38 \pm 15.03$ \\
TV-A (cm/s) & $49.74 \pm 15.3$ & $61.64 \pm 15.03^{*}$ & $58.56 \pm 18.27^{*}$ \\
TV-E/A & $1.31 \pm 0.24$ & $1.00 \pm 0.3^{*}$ & $1.05 \pm 0.31^{*}$ \\
IVRT (ms) & $59.32 \pm 10.43$ & $80.22 \pm 23.52^{*}$ & $83.06 \pm 18.34^{*}$ \\
DT (ms) & $188.94 \pm 26.22$ & $165.33 \pm 49.8^{*}$ & $180.64 \pm 40.9^{\S}$ \\
TEI index & $0.43 \pm 0.07$ & $0.59 \pm 0.11^{*}$ & $0.60 \pm 0.10^{*}$ \\
\hline
\end{tabular}

RVSP: Right ventricular systolic pressure, TV-E: Tricuspid valve early diastolic velocity, TV-A: TV late diastolic velocity, IVRT: Iso-volemic relaxation time,

DT: Deceleration time, COPD: Chronic obstructive pulmonary disease, ${ }^{*} \mathrm{p}<0.05$ vs. controls, ${ }^{\S} \mathrm{p}<0.05$ vs. A/E of COPD group 
Table 5: Tissue Doppler imaging

\begin{tabular}{llll}
\hline Echo parameters & Controls & COPD with A/E state & Remission state after A/E event \\
\hline RVE (m/s) & $0.13 \pm 0.03$ & $0.11 \pm 0.02^{*}$ & $0.11 \pm 0.03^{*}$ \\
RVA (m/s) & $0.15 \pm 0.05$ & $0.20 \pm 0.05^{*}$ & $0.18 \pm 0.04^{*}$ \\
RVS (m/s) & $0.18 \pm 0.02$ & $0.14 \pm 0.03^{*}$ & $0.15 \pm 0.04^{*}$ \\
IVSE (m/s) & $0.11 \pm 0.02$ & $0.08 \pm 0.02^{*}$ & $0.08 \pm 0.02^{*}$ \\
IVSA (m/s) & $0.09 \pm 0.02$ & $0.10 \pm 0.02^{*}$ & $0.10 \pm 0.02^{*}$ \\
IVSS (m/s) & $0.09 \pm 0.01$ & $0.08 \pm 0.01$ & $0.08 \pm 0.02$ \\
\hline
\end{tabular}

RV: Right ventricle, IVS: Inter ventricular septum, E: Early diastolic tissue annular velocity, A: Late diastolic tissue annular velocity, S: Systolic annular velocity, COPD: Chronic obstructive pulmonary disease, ${ }^{*} \mathrm{p}<0.05$ vs. controls, ${ }^{\circledR} \mathrm{p}<0.05 \mathrm{vs}$. A/E of COPD group

Table 6: RV deformation imaging

\begin{tabular}{llll}
\hline Echo parameters & Controls & COPD with A/E state & Remission state after A/E event \\
\hline RVSI (\%) & $-31.16 \pm 3.74$ & $-24.96 \pm 4.8^{*}$ & $-26.01 \pm 1.06^{*}$ \\
RV SSR (/s) & $-2.8 \pm 0.64$ & $-1.85 \pm 0.46^{*}$ & $-1.81 \pm 0.58^{*}$ \\
RV ESR (/s) & $2.54 \pm 0.64$ & $1.83 \pm 0.52^{*}$ & $2.07 \pm 1.5^{*}$ \\
RV LSR (/s) & $2.41 \pm 0.76$ & $2.34 \pm 0.67$ & $2.24 \pm 0.66$ \\
IVS SI (\%) & $-24.62 \pm 1.78$ & $-14.80 \pm 1.97^{*}$ & $-21.01 \pm 3.65^{*} \delta$ \\
IVS SSR (/s) & $-1.57 \pm 0.26$ & $-1.29 \pm 0.36^{*}$ & $-1.36 \pm 0.32^{*}$ \\
IVS ESR (/s) & $1.7 \pm 0.52$ & $1.55 \pm 0.5$ & $1.55 \pm 0.44$ \\
IVS LSR (/s) & $1.81 \pm 0.43$ & $1.63 \pm 0.48$ & $1.77 \pm 0.59$ \\
\hline
\end{tabular}

RV: Right ventricle, IVS: Inter ventricular septum, SI: Systolic strain imaging, SSR: Systolic strain rate, ESR: Early diastolic strain rate, LSR: Late diastolic strain rate, COPD: Chronic obstructive pulmonary disease, ${ }^{*} \mathrm{p}<0.05$ vs. controls, ${ }^{\circledR} \mathrm{p}<0.05 \mathrm{vs}$. A/E of COPD group

distribution of the given variable. The RV strain as measured by tissue Doppler technique showed a positive correlation with FEV1/VC ratio though was not statistically significant (Table 7 and Fig. 2)

SSR of RV also did not show significant linear correlation with the values of FEV1/VC (Table 8 and Fig. 3).

The measure of global RV function, TEI index was well correlated with the value of peak systolic strain of RV and was statistically significant with negative linear correlation (Table 9 and Fig. 4).

However, the FEV1/VC did not show significant linear correlation with systolic tissue annular velocity of RV (Fig. 5).

\section{DISCUSSION}

This study shows that, in COPD patients, tissue Doppler parameters can detect RV dysfunction that is complementary to conventional echocardiographic indices and they correlate with severity of pulmonary disease. This study showed a significant increase in RV thickness and the chamber dimensions in patients with COPD when compared to controls. RV hypertrophy and dilatation that occurs in COPD patients is a beneficial adaptation allowing the ventricle to cope with an increased afterload and maintain a normal cardiac output. Progressive RV dysfunction is associated with limited peripheral oxygen delivery and exercise capacity and has an important bearing on prognosis that is independent of other factors, such as severity of airflow obstruction, and reflects the interrelationship with deranged pulmonary hemodynamics.

Tricuspid late diastolic A wave, IVRT, TEI index values were significantly increased in COPD patients whereas E/A ratio, deceleration time were significantly reduced in COPD with A/E group suggesting the presence of RV diastolic dysfunction in the study group. Tissue annular velocity showed significant reduction in tissue annular motion including RVED and RVES velocities as well as early diastolic annular velocity of IVS. This shows underlying impairment in RV diastolic as well as systolic function in COPD patients. Similar findings were revealed in the study of Vitarelli et al. [27].

\section{RV deformation by TDI}

An advantage of using TDI to assess RV function is that measurement is independent of geometric assumptions and endocardial border tracing. However, one limitation of TDI is that it does not distinguish between active and passive wall motion, and this has led to the development of
Table 7: RV strain as measured by tissue Doppler technique

\begin{tabular}{lll}
\hline RV strain & FEV1_VC_B & SI_RV_AVG_B \\
\hline FEV1_VC_B & & \\
$\quad$ Pearson correlation & 1 & 0.226 \\
$\quad \begin{array}{l}\text { Significant (one-tailed) } \\
\text { n }\end{array}$ & 0.093 & \\
SI_RV_AVG_B & 36 & 36 \\
$\quad \begin{array}{l}\text { Pearson correlation } \\
\text { Significant }\end{array}$ & 0.226 & 1 \\
$\quad$ (one-tailed) & & 0.093 \\
n & 36 & 36 \\
\hline
\end{tabular}

FEV1/VC_B: Forced expiratory volume in one second per vital capacity at baseline, SI: Strain imaging, RV: Right ventricle

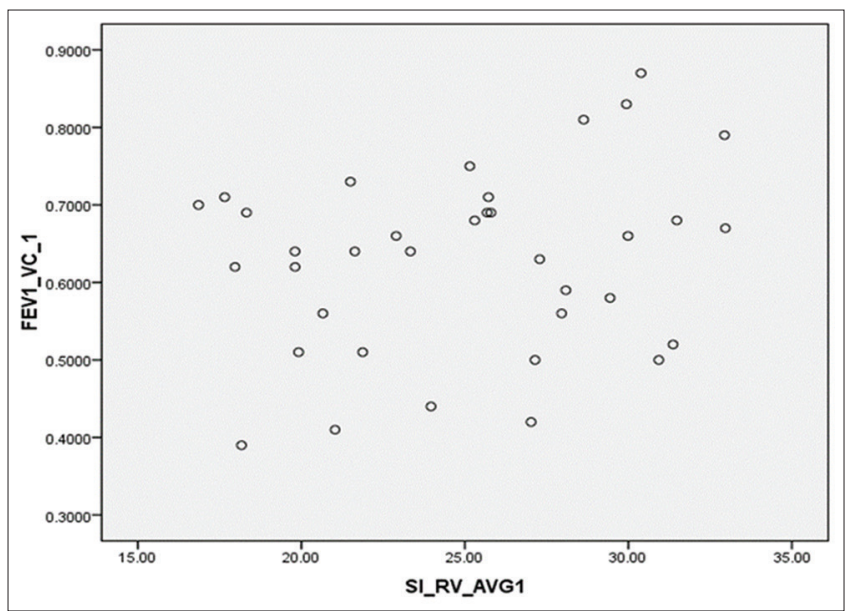

Fig. 2: The right ventricular strain as measured by tissue Doppler technique, FEV1/VC_B: Forced expiratory volume in 1 second/vital capacity at baseline, SI: Strain imaging

the TDI-derived modalities of strain and SR that measure the rate of regional myocardial deformation.

This study describes the characteristics of ultrasound-based regional deformation indices in patients with acute exacerbation of COPD and 
Table 8: Systolic strain of RV

\begin{tabular}{lll}
\hline RV strain & FEV1_VC_B & SSR_RV_AVG_B \\
\hline FEV1_VC_B & & \\
$\quad$ Pearson correlation & 1 & 0.097 \\
$\quad$ Significant (one-tailed) & 0.286 & \\
n & 36 & 36 \\
SSR_RV_AVG_B & & \\
$\quad$ Pearson correlation & 0.097 & 1 \\
Significant (one-tailed) & 0.286 & \\
n & 36 & 36 \\
\hline
\end{tabular}

FEV1/VC_B: Forced expiratory volume in one second per vital capacity at

baseline, SSR: Systolic strain rate, RV: Right ventricle

Table 9: RV global function measure

\begin{tabular}{lll}
\hline RV strain & TEI_B & SI_RV_AVG_B \\
\hline FEV1_VC_B & & \\
$\quad$ Pearson correlation & 1 & -0.533 \\
Significant (one-tailed) & 0.000 & 70 \\
n & 70 & \\
SI_RV_AVG_B & & 1 \\
Pearson correlation & -0.533 & \\
Significant (one-tailed) & 0.000 & 70 \\
n & 70 & \\
\hline
\end{tabular}

TEI_B: Tei index of RV at baseline, SI: Strain imaging, RV: Right ventricle

their correlation with pulmonary function tests. In the patients of the current study, reduced regional long-axis shortening was observed in all segments of the RVFW. Although the myocardial deformation rate and TEI index are two different measurements, this study shows a significant correlation between the peak systolic strain and the RV TEI index. Furthermore, in contrast to other ultrasound-based methods of quantifying global RV function, such as the TEI index, SRI potentially allows the quantification of longitudinally systolic and diastolic function for each segment of the RV.

The non-homogeneity in longitudinal RV function observed in the patients of the current study is presumably a result of the complex anatomy of the RV that is comprised three morphologically distinct units: A smooth inlet portion, a tubular infundibulum, and a more trabeculated apical portion. As a result of their different muscular arrangements, each could respond differently to both changes in preload and afterload, and fiber stretch as a result of dilatation. When the overall RV function is taken into account, the inlet area of the RV chamber has a greater contribution compared with the trabecular region and the infundibulum. This may explain the nonhomogeneous decrease in systolic deformation that occurs in COPD patients during times of worsening hypoxia, and hence increased afterload stress.

This study demonstrates the utility of SRI parameters for the evaluation of RV function in COPD patients. The new echo technologies show impairment of RV function that is related to pulmonary hypertension and severity of pulmonary disease and are more sensitive than conventional echocardiographic parameters. SRI indices could provide clinically relevant information on regional changes in RV function in chronic pulmonary disease and contribute toward an understanding of how the differing structural components of the RV deteriorate after increasing afterload, and how they recover after appropriate therapy.

Our findings of diastolic dysfunction in patients with COPD, indicated by an increased IVRT and E/Ea, are supported by two recent studies $[27,28]$.

Impaired RV systolic function, and in particular, reduced contractility as indicated by a lower FW strain, SR was present in the patients

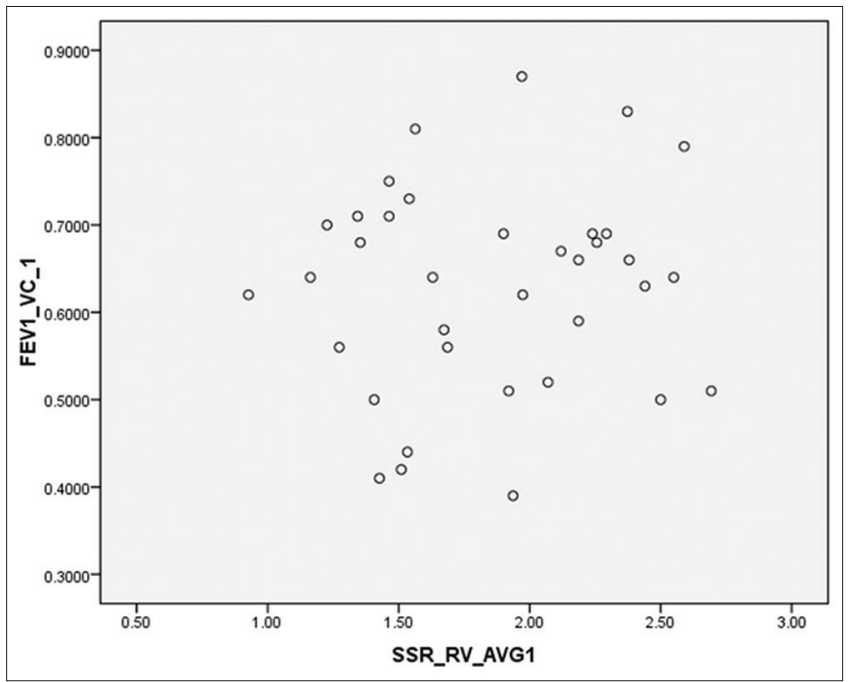

Fig. 3: Systolic strain rate of right ventricle, FEV1/VC_B: Forced expiratory volume in 1 second/vital capacity at baseline, SSR: Systolic strain rate, RV: Right ventricle

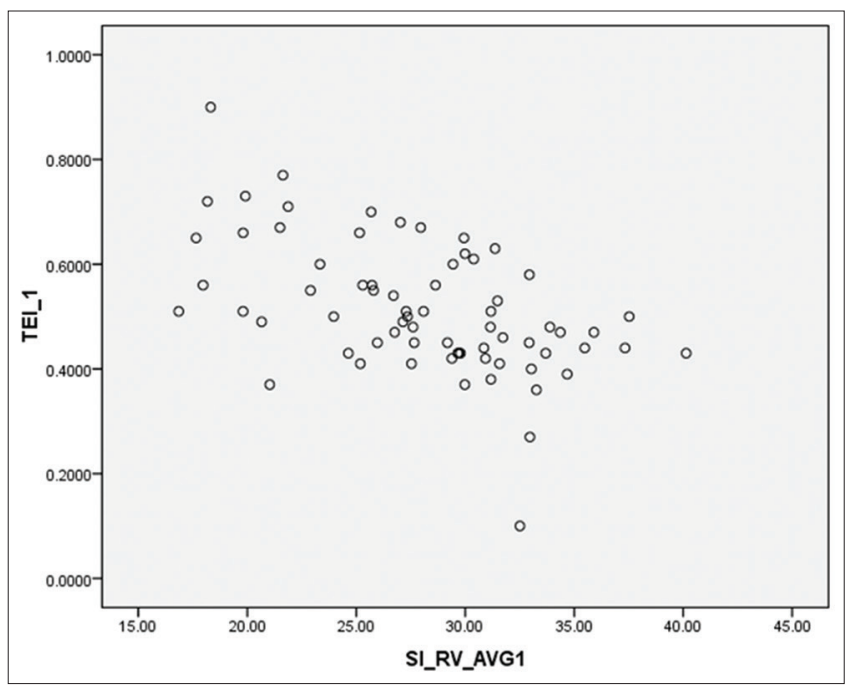

Fig. 4: Right ventricle global function measure, TEI B: Tei index of RV at baseline, SI: strain imaging, RV: Right ventricle

including those with even post exacerbation event at the remission state, when compared with controls. None of our patients had clinical evidence of cor pulmonale and estimation of PA pressure using the tricuspid regurgitant (TR) jet did not separate patient at remission state and control groups; however, changes in surrogate indices of PA hypertension, including RVFW myocardial relaxation time, TEI index, indicated RV functional changes consistent with a degree of increased pulmonary vascular resistance and increased afterload in patients with both mild and severe lung disease. This confirms the findings of RV dysfunction without clinical evidence of cor pulmonale in patients with COPD where such features were mainly associated with pressures $>35 \mathrm{mmHg}$, though, similar to our study, systolic strain and SR reductions were present in patients with pressures $<35 \mathrm{mmHg}$ [12].

\section{RV function at remission state}

This study showed a significant decline in tissue Doppler parameters including annular velocity, strain, and SR variables at remission state from A/E of COPD event when compared with controls even when PA pressure seem to be normal as assessed by TR jet. This finding is supported by the study conducted by Hilde et al. [29] where they found 


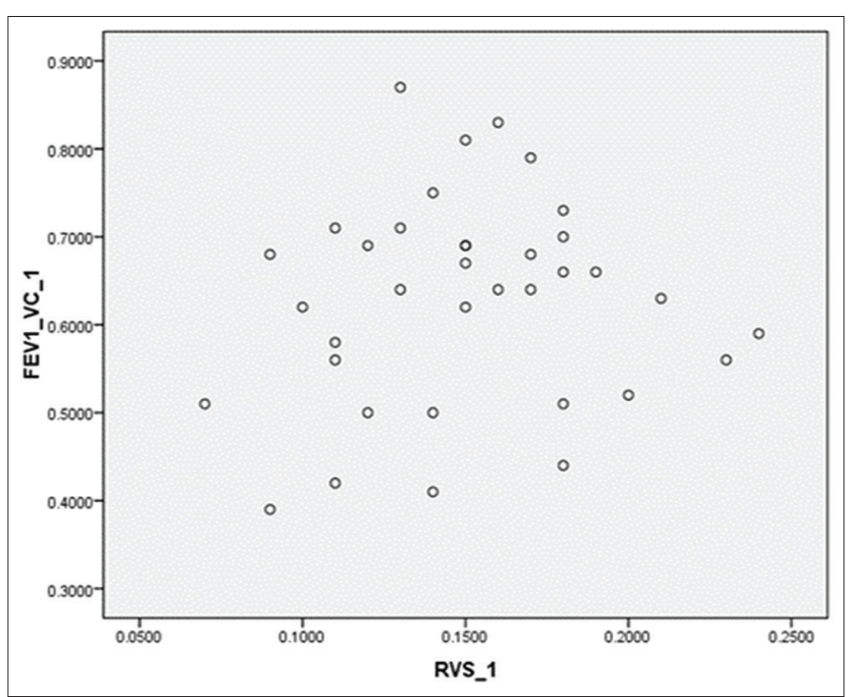

Fig. 5: Right ventricular global function measure, FEV1/VC_B:

Forced expiratory volume in 1 second/vital capacity at baseline, RVS: Right ventricular systolic tissue annular velocity

that the COPD patients with relatively normal PA pressure possessed subclinical RV dysfunction. Thus, the study reveals, permanent changes that result due to acute exacerbation event remain unaltered in the remission state even when PA pressure drops to a normal range. In a study performed by Vitarelli et al. concluded that in COPD patients, SRI parameters can determine RV dysfunction that is complementary to conventional echocardiographic indices and is correlated with pulmonary hypertension and respiratory function tests [12], which can support similar finding in this study.

\section{CONCLUSION}

- Acute exacerbation of COPD causes worsening of RV function

- Impaired RV function remain unaltered at remission state as assessed by tissue Doppler parameters such as annular velocities, strain and SR even when PA pressure falls to normal range

- Because RV dysfunction has a negative impact on prognosis, assessment of RV function is very important in patients with A/E of COPD as well as at remission state

- Noninvasive evaluation of RV longitudinal function such as strain and SR by TDI has gained importance because it is easy to obtain, quantitative and reproducible.

\section{ACKNOWLEDGMENTS}

The authors would like to acknowledge the role of Manipal University in encouraging us and allowing the use of their resources for the conduct of this study.

\section{REFERENCES}

1. Murray CJ, Lopez AD. Global mortality, disability, and the contribution of risk factors: Global Burden of Disease Study. Lancet 1997;349(9603):1436-42.

2. Murray CJ, Lopez AD. Regional patterns of disability-free life expectancy and disability-adjusted life expectancy: Global burden of disease study. Lancet 1997;349(9062):1347-52.

3. Bolton CE, Ionescu AA, Shiels KM, Pettit RJ, Edwards PH, Stone MD, et al. Associated loss of fat-free mass and bone mineral density in chronic obstructive pulmonary disease. Am J Respir Crit Care Med 2004;170(12):1286-93.

4. Sin DD, Man SF. Chronic obstructive pulmonary disease as a risk factor for cardiovascular morbidity and mortality. Proc Am Thorac Soc 2005;2(1):8-11.

5. Sin DD, Wu L, Man SF. The relationship between reduced lung function and cardiovascular mortality: A population-based study and a systematic review of the literature. Chest 2005;127(6):1952-9.
6. Weitzenblum E. Chronic cor pulmonale. Heart 2003;89(2):225-30.

7. Curkendall SM, DeLuise C, Jones JK, Lanes S, Stang MR, Goehring E Jr, et al. Cardiovascular disease in patients with chronic obstructive pulmonary disease, Saskatchewan Canada cardiovascular disease in COPD patients. Ann Epidemiol 2006;16(1):63-70.

8. Rutten FH, Cramer MJ, Grobbee DE, Sachs AP, Kirkels JH, Lammers JW, et al. Unrecognized heart failure in elderly patients with stable chronic obstructive pulmonary disease. Eur Heart J 2005;26(18):1887-94.

9. Weber T, Auer J, O'Rourke MF, Kvas E, Lassnig E, Lamm G, et al. Increased arterial wave reflections predict severe cardiovascular events in patients undergoing percutaneous coronary interventions. Eur Heart J 2005;26(24):2657-63.

10. Mattace-Raso FU, van der Cammen TJ, Hofman A, van Popele NM, Bos ML, Schalekamp MA, et al. Arterial stiffness and risk of coronary heart disease and stroke: The Rotterdam study. Circulation 2006;113(5):657-63.

11. Sabit R, Bolton CE, Edwards PH, Pettit RJ, Evans WD, McEniery CM, et al. Arterial stiffness and osteoporosis in chronic obstructive pulmonary disease. Am J Respir Crit Care Med 2007:175(12):1259-65.

12. Vitarelli A, Conde Y, Cimino E, Stellato S, D'Orazio S, D'Angeli I, et al. Assessment of right ventricular function by strain rate imaging in chronic obstructive pulmonary disease. Eur Respir J 2006;27(2):268-75.

13. López-Candales A, Rajagopalan N, Dohi K, Gulyasy B, Edelman K, Bazaz R. Abnormal right ventricular myocardial strain generation in mild pulmonary hypertension. Echocardiography 2007;24(6):615-22.

14. Rabe KF, Hurd S, Anzueto A, Barnes PJ, Buist SA, Calverley P, et al. Global strategy for the diagnosis, management, and prevention of chronic obstructive pulmonary disease: GOLD executive summary. Am J Respir Crit Care Med 2007;176(6):532-55.

15. Redfield MM, Jacobsen SJ, Borlaug BA, Rodeheffer RJ, Kass DA. Age- and gender-related ventricular-vascular stiffening: A community-based study. Circulation 2005;112(5):2254-62.

16. Sutton-Tyrrell K, Najjar SS, Boudreau RM, Venkitachalam L, Kupelian V, Simonsick EM, et al. Elevated aortic pulse wave velocity, a marker of arterial stiffness, predicts cardiovascular events in wellfunctioning older adults. Circulation 2005;111(25):3384-90.

17. Fernandes VR, Polak JF, Cheng S, Rosen BD, Carvalho B, Nasir K, et al. Arterial stiffness is associated with regional ventricular systolic and diastolic dysfunction: The multi-ethnic study of atherosclerosis. Arterioscler Thromb Vasc Biol 2008;28(1):194-201

18. Mottram PM, Haluska BA, Leano R, Carlier S, Case C, Marwick TH. Relation of arterial stiffness to diastolic dysfunction in hypertensive heart disease. Heart 2005;91(12):1551-6.

19. Hundley WG, Kitzman DW, Morgan TM, Hamilton CA, Darty SN, Stewart KP, et al. Cardiac cycle-dependent changes in aortic area and distensibility are reduced in older patients with isolated diastolic heart failure and correlate with exercise intolerance. J Am Coll Cardiol 2001;38(3):796-802

20. France AJ, Prescott RJ, Biernacki W, Muir AL, MacNee W. Does right ventricular function predict survival in patients with chronic obstructive lung disease? Thorax 1988:43:621-6.

21. Burgess MI, Mogulkoc N, Bright-Thomas RJ, Bishop P, Egan JJ, Ray SG. Comparison of echocardiographic markers of right ventricular function in determining prognosis in chronic pulmonary disease. J Am Soc Echocardiogr 2002;15:633-9.

22. Vestbo J, Hurd SS, Agustí AG, Jones PW, Vogelmeier C, Anzueto A, et al. Global strategy for the diagnosis, management, and prevention of chronic obstructive pulmonary disease: GOLD executive summary. Am J Respir Crit Care Med 2013;187(4):347-65.

23. Terzano C, Romani S, Paone G, Conti V, Oriolo F. COPD and thyroid dysfunctions. Lung 2014;192(1):103-9.

24. Schenk P, Globits S, Koller J, Brunner C, Artemiou O, Klepetko W, et al. Accuracy of echocardiographic right ventricular parameters in patients with different end-stage lung diseases prior to lung transplantation. J Heart Lung Transplant 2000;19(2):145-54.

25. Bommer W, Weinert L, Neumann A, Neef J, Mason DT, DeMaria A. Determination of right atrial and right ventricular size by twodimensional echocardiography. Circulation 1979;60:91-100.

26. Kukulski T, Hübbert L, Arnold M, Wranne B, Hatle L, Sutherland GR. Normal regional right ventricular function and its change with age: A Doppler myocardial imaging study. J Am Soc Echocardiogr 2000;13(3):194-204

27. Weidemann F, Eyskens B, Jamal F, Mertens L, Kowalski M, D’Hooge J, et al. Quantification of regional left and right ventricular radial and longitudinal function in healthy children using ultrasound-based strain 
rate and strain imaging. J Am Soc Echocardiogr 2002;15(1):20-8.

28. Dambrauskaite V, Herbots L, Claus P, Verleden G, Van Raemdonck D, Delcroix $\mathrm{M}$, et al. Differential changes in regional right ventricular function before and after a bilateral lung transplantation: An ultrasonic strain and strain rate study. J Am Soc Echocardiogr
$2003 ; 16(5): 432-6$

29. Hilde JM, Skjørten I, Grøtta OJ, Hansteen V, Melsom MN, Hisdal J, et al. Right ventricular dysfunction and remodeling in chronic obstructive pulmonary disease without pulmonary hypertension. J Am Coll Cardiol 2013;62(12):1103-11. 